\title{
Evolución del empleo y del paro de las mujeres inmigrantes en el mercado de trabajo español. El impacto de la actual crisis económica ${ }^{*}$
}

\author{
Jacobo MUÑOZ COMET \\ Dep. Sociología II \\ Universidad Nacional de Educación a Distancia \\ jmcomet@bec.uned.es
}

Recibido: 31-01-2011

Aceptado: 19-09-2011

\begin{abstract}
RESUMEN
Este artículo aborda el impacto de la actual crisis económica sobre el nivel de ocupación de las mujeres inmigrantes. Para ello se utilizan datos de la Encuesta de Población Activa desde 2000 hasta 2010. Los resultados muestran que las mujeres extranjeras están soportando mejor el efecto de la recesión que sus connacionales, cuyas tasas de paro han crecido en mayor medida. Además, el capital humano de las mujeres inmigrantes ofrece una mayor resistencia frente a la caída de la tasa de empleo que el de los inmigrantes varones. A partir de la recesión, el nivel de ocupación de las mujeres españolas es de todos los grupos el que experimenta pérdidas más moderadas, aunque en su caso un nivel educativo alto no implica un descenso más suave de la tasa de empleo. Por otra parte, un mayor tiempo en el mercado laboral sólo parece ser un factor de protección en el caso de las mujeres españolas.
\end{abstract}

Palabras clave: ocupación; segmentación; capital humano; nivel educativo; tiempo en el mercado de trabajo

Evolution of employment of female immigrants in the Spanish labour market. The impact of the current financial crisis

\begin{abstract}
This paper explores the impact of the current financial crisis on employment of female immigrants by conducting empirical analysis based on the Spanish Labour Force Survey, from 2000 until 2010. Results show that female immigrants are less affected by the recession in comparison to male immigrants. At the same time, human capital of female immigrants is more effective against the employment fall in comparison to male immigrants, of which the unemployment rate shows a higher growth. As for Spanish women, employment rates show the smallest drop since the recession, having the same effect on high as well as low educated women. Finally, time in the labour market only has a positive impact on prospects of female natives.
\end{abstract}

Key words: occupation; segmentation; human capital; educational level; time in the labour market

* Este trabajo se enmarca dentro del proyecto de $\mathrm{I}+\mathrm{D}+\mathrm{i}$ "Estratificación ocupacional y rendimiento de la formación en España: ajuste educación-empleo, inmigración y jubilación" (CSO2010-21004), desarrollado en el Departamento de Sociología II de la UNED. 


\section{REFERENCIA NORMALIZADA}

Muñoz Comet, J. (2012). Evolución del empleo y del paro de las mujeres inmigrantes en el mercado de trabajo español. El impacto de la actual crisis económica. Cuadernos de Relaciones Laborales. Vol. 30, núm.1, p. 115-137.

SUMARIO: Introducción. 1. Marco teórico. 2. Hipótesis. 3. Datos, variables y técnicas. 4. Resultados. 5. Conclusiones. Bibliografía

\section{Introducción}

Antes de que la actual crisis económica irrumpiese, el mercado de trabajo español estaba experimentando uno de los periodos más extraordinarios de toda su historia. Tras la recesión sufrida a principios de la década de los noventa, el ritmo de creación de empleo vivido en los años posteriores alcanzó unos niveles sin precedentes, pasando de algo más de 12 millones de ocupados en 1994 a unos 20 millones en 2008. El ciclo expansivo de aquellos años coincide con uno de los fenómenos sociales más importantes ocurrido en las últimas décadas, la explosión migratoria de los flujos de entrada. España, que venía siendo históricamente un país de emigrantes, ha pasado de tener 500.000 inmigrantes en 1996 a más de 5,7 millones en 2011, es decir, un $12,2 \%$ de las personas residentes en el país, según datos del Padrón $\mathrm{Mu}$ nicipal ${ }^{1}$.

El periodo de bonanza económica experimentado durante algo más de una década se ha visto interrumpido, desde finales de 2007, por la recesión económica. El impacto de la crisis está azotando al conjunto de la población, sin embargo, algunos colectivos están siendo más perjudicados que otros, entre ellos la población inmigrante. Diversos informes señalan que durante las fases negativas del ciclo económico, los trabajadores extranjeros suelen ser uno de los grupos más expuestos a la pérdida de empleo, debido tanto a su perfil sociodemográfico como al tipo de puestos de trabajo que suelen ocupar (Kochhar, 2003, 2008; Pajares, 2009; Papademetrou y Terrazas, 2009; Garson y Dumont, 2009).

Pese a la juventud del fenómeno, la inserción laboral de los inmigrantes en España ha sido abordada desde diferentes puntos de vista. Uno de los aspectos más

\footnotetext{
${ }^{1}$ En este trabajo se va a estudiar a la población extranjera, es decir, aquellas personas que no poseen la nacionalidad española. Para evitar posibles sesgos debido a la llegada de oriundos y a la intensidad del proceso de naturalización, las personas con nacionalidad española se van a separar entre las nacidas en España y aquellas otras nacidas en el extranjero o con doble nacionalidad (una de ellas la española). Asimismo, a lo largo del artículo se va emplear el término inmigrante como sinónimo de extranjero, aunque siempre se estará haciendo referencia a la condición de no poseer nacionalidad española, y no al lugar de nacimiento.
} 
destacados en diversos trabajos es la segregación sectorial que sufren los trabajadores extranjeros. En los años previos a la actual crisis económica las tasas de empleo y paro de autóctonos y de inmigrantes eran bastante parecidas con la excepción del colectivo africano (Garrido, 2008). Sin embargo, los puestos de trabajo de la gran mayoría de los inmigrantes pertenecían a la parte más baja de la estructura ocupacional. Este grupo de ocupaciones ha experimentado desde la mitad de los años noventa un fuerte crecimiento en términos absolutos, aunque no en términos relativos (Bernardi y Garrido, 2008). Al finalizar el periodo de expansión, la situación laboral de los inmigrantes económicos se caracterizaba por una alta temporalidad del empleo, baja cualificación y escasas posibilidades de promoción independientemente del tiempo en el mercado de trabajo (Iglesias y Llorente, 2006; Garrido y Miyar, 2008; Cebolla y González, 2009)2.

La destrucción de más de dos millones de puestos de trabajo se ha producido, en buena parte, en los nichos laborales donde la mayoría de los inmigrantes estaban concentrados antes de la crisis (Garrido, Miyar y Muñoz, 2010; Garrido y Rodríguez, 2011). El objetivo de este artículo es conocer cómo ha afectado el cambio de ciclo sobre la ocupación de las mujeres inmigrantes en comparación con la de los varones de su misma nacionalidad. En concreto, se quiere estudiar si el capital humano de ambos grupos ofrece la misma resistencia frente a la crisis en un mercado de trabajo segmentado que, a priori, afecta a mujeres y varones inmigrantes por igual. Para ello se realiza un análisis descriptivo de sus tasas de empleo y de paro a lo largo de toda la primera década del siglo XXI, teniendo en cuenta el nivel educativo y el tiempo en el mercado de trabajo ${ }^{3}$. Además de comparar por sexo dentro de cada nacionalidad, también se estudiará la evolución experimentada por las españolas en comparación con la de las mujeres extranjeras.

El artículo está estructurado en cinco secciones. En la primera de ellas se desarrolla el marco teórico a partir del cual se definen las hipótesis del estudio, las cuales se presentan en el segundo apartado. En la tercera sección se explican los datos con los que se va a trabajar, así como las variables construidas y las técnicas utilizadas. Finalmente, en las dos últimas secciones se exponen los resultados y las conclusiones del trabajo, respectivamente.

${ }^{2}$ El término "inmigrantes económicos" es utilizado para referirse a aquellos extranjeros que proceden de naciones con una renta inferior a la del país de acogida.

${ }^{3}$ El tiempo en el mercado de trabajo hace referencia a la experiencia laboral potencial, sin distinguir entre periodos de ocupación o desocupación. También se utilizará el término "edad laboral". 


\section{Marco teórico}

El término "mercado dual" se utiliza para hacer referencia a aquellos mercados que están divididos en, al menos, dos segmentos, cuyos mecanismos de funcionamiento difieren entre sí. El segmento primario se caracteriza por ofrecer unos puestos de trabajo con salarios elevados, buenas condiciones de trabajo con posibilidad de ascenso, equidad y procedimientos estándar en la aplicación de las normas laborales y, sobre todo, estabilidad en el empleo. El segmento secundario se caracteriza por todo lo contrario: puestos de trabajo peor pagados, escasas posibilidades de promoción, relaciones muy personalizadas y por ello caracterizadas por favoritismos y arbitrariedades, y principalmente, alta inestabilidad y rotación en los puestos de trabajo (Piore, 1999). Los empleos dentro del segmento secundario son mucho más vulnerables a los cambios en la coyuntura económica y, por tanto, son más sensibles a las fases recesivas.

El mercado laboral español muestra rasgos propios de un mercado dual. Por ejemplo, los mecanismos que determinan los salarios en uno y otro segmento son diferentes. En el segmento primario la influencia del capital humano, en concreto la formación y la antigüedad en el puesto de trabajo, es mayor que en el segmento secundario (Huguet, 1996). En este marco hay que contextualizar la segregación laboral de los inmigrantes. Los trabajadores extranjeros se concentran, básicamente, en cinco ramas de actividad: servicio doméstico, agricultura, hostelería, comercio al por menor y construcción. El estatus que tiene un trabajador en el mercado de trabajo está condicionado por su posición en el sistema de reproducción social tanto en el ámbito familiar como en la estructura de clase-, lo cual determina su "nivel de aceptación" de las condiciones laborales (Cachón, 1997). La posición que ocupan los trabajadores inmigrantes y españoles en este sistema y, por ello, sus distintos niveles de aceptación les hacen divergir en su idea de un trabajo "socialmente inaceptable". No obstante, la concentración que han experimentado los trabajadores extranjeros en los puestos de trabajo de menor cualificación también puede estar relacionada con el vuelco educacional vivido en España en las últimas décadas:

Se puede afirmar que buena parte [de los inmigrantes] ha venido a cubrir unos puestos de trabajo descualificados cuya ocupación se había problematizado, no tanto por el rechazo de los potenciales trabajadores españoles de bajo nivel formativo, sino más bien por la disminución radical del tamaño de ese colectivo en la última década. (Garrido, 2008:142)

La inserción laboral en un mercado de trabajo puede ser explicada en términos de capital humano. La clave estaría en que los individuos acceden al mercado laboral con diferentes niveles de cualificación, no solamente debido a sus distintas capacidades innatas, sino también a las diferencias entre unos y otros debido al tiempo que han dedicado a formarse, es decir, a la inversión en capital humano (Becker, 1999). Así la oferta de trabajo no sólo se basa en la cantidad de trabajo que 
un individuo puede producir, sino también en la calidad que es capaz de ofrecer. Además de la ocupación, esta teoría explica el funcionamiento de otros procesos del mercado de trabajo. Por ejemplo, las diferencias salariales entre individuos responderían al grado de inversión en capital humano: los más cualificados son quienes obtienen unos sueldos más elevados. En el otro extremo, quienes reciben remuneraciones más bajas o se encuentran sin empleo encontrarían el origen de su situación en un insuficiente nivel de capital humano.

Existe abundante literatura acerca del proceso de integración laboral de los extranjeros en el país de destino. Uno de los artículos más citados es el de Barry R. Chiswick (1978), quien utiliza la perspectiva del capital humano para explicar las diferencias salariales de inmigrantes y nativos. Aunque a su llegada reciben un sueldo inferior, con el aumento de la experiencia laboral los salarios de los inmigrantes van creciendo, hasta igualarse con los de los autóctonos tras 10 o 15 años de residencia en el país de acogida. Chiswick interpreta sus resultados desde un enfoque neoclásico, por el cual los inmigrantes recién llegados poseen un capital humano poco específico para alcanzar una buena posición en el nuevo mercado de trabajo. El aprendizaje durante los primeros años de residencia (idioma, nueva formación, experiencia en puestos de trabajo...) incrementa su capital humano específico del país de destino, de forma que la brecha respecto a los trabajadores nativos se reduce o incluso termina desapareciendo. Algunos estudios más recientes centrados en las diferencias salariales en distintos países confirman esta tesis (Dustmann, 1994; Skyt y otros, 2001; Lam y Liu, 2002; Dustmann y Fabbri, 2003; Mato y Gutiérrez, 2010).

Por otra parte, la asimilación laboral de los inmigrantes puede verse afectada por un cambio de ciclo económico. Boisjoly y Duncan (1994) estudian el impacto de la recesión de principios de los noventa en Estados Unidos. Los resultados señalan que los trabajadores de origen hispano, particularmente los inmigrantes, tienen más probabilidades de perder su empleo durante la crisis que el resto de trabajadores. Algunas diferencias sociodemográficas, principalmente unos niveles de educación escolar más bajos, explicarían gran parte de esta brecha. En comparación con el resto, los hispanos suelen ser más jóvenes, poseer un nivel educativo más bajo y acumular menos experiencia laboral. De este modo, el tipo de ocupaciones y de industrias a los que pueden acceder les colocan en una posición especialmente vulnerable en periodos de recesión. A conclusiones similares llega Kogan (2004), quien encuentra que el mayor riesgo al desempleo de los trabajadores inmigrantes reside en factores relacionados con el capital humano, pero sobre todo por la posición en el mercado laboral, generalmente sobrerrepresentados en las ocupaciones de menor cualificación.

Otros trabajos apoyan la importancia del nivel educativo y de la experiencia laboral en la probabilidad de estar empleado. La influencia positiva de estos dos factores suele ser mayor entre nativos que entre extranjeros (Chiswick, Cohen y Zach, 1997). El motivo estaría en que la formación y la experiencia laboral de los inmigrantes fueron adquiridas antes de emigrar. El capital humano acumulado en el periodo pre-migratorio tiene un escaso rendimiento en el mercado de trabajo del país de acogida. Es decir, no siempre es transferible de un país a otro, lo cual obliga 
a adquirir un capital humano específico del país al que se emigra (Chiswick 1978, 1979; Friedberg, 2000; Weiss, Sauer y Gotlibovsky, 2003). Algo importante del estudio de Chiswick, Cohen y Zach es que no hallan ninguna relación entre acceder al mercado laboral en un periodo con alta tasa de paro - en fase recesiva- y una vida laboral posterior con menos oportunidades de empleo.

En España, Amuedo-Dorantes y De la Rica (2006) encuentran que los trabajadores inmigrantes tienen menos probabilidades de estar empleados que los nativos. Sin embargo, esta brecha se explica en buena parte tras controlar la edad y el nivel educativo. Al hacerlo, las diferencias entre inmigrantes y autóctonos prácticamente desaparecen al segundo año de residencia, aunque el proceso de asimilación difiere dependiendo de la nacionalidad. Por ejemplo, los europeos de fuera de la UE15 son los que más rápido convergen con los españoles, al contrario que los africanos, cuya asimilación se produce a un ritmo más lento. Los resultados del trabajo también muestran que las diferencias entre extranjeros y autóctonos se reducen principalmente durante el primer año de residencia en España. Hay que señalar que los datos que utilizan las autoras hacen referencia a los años 2001 y 2002, cuando la intensa llegada de inmigrantes acababa de empezar.

Fernández y Ortega (2008) también observan que el capital humano de los inmigrantes adquirido en su país de origen (nivel educativo y experiencia laboral) ofrece un bajo rendimiento en el mercado de trabajo del país de destino. Respecto a las tasas de desempleo - más elevadas entre los extranjeros-, la brecha que existe al inicio entre inmigrantes y nativos se reduce con el paso de los años, en la misma línea que el trabajo de Chiswick et al. (1997). Las autoras señalan, por otra parte, que los inmigrantes son más sensibles que los nativos a los cambios del ciclo económico. Así, prevén que los extranjeros tengan más probabilidades de sufrir desempleo durante un periodo de recesión que los autóctonos. Por último, Bernardi, Garrido y Miyar (2011) encuentran de nuevo evidencias de que los inmigrantes no tienen desventajas en comparación con los españoles en el riesgo de sufrir desempleo. Esto es así una vez controlados algunos factores sociodemográficos - como el nivel educativo- $-\mathrm{y}$, sobre todo, después de tener en cuenta el tiempo en el mercado de trabajo.

\section{Hipótesis}

El estudio de la evolución de la ocupación de las mujeres inmigrantes se va a llevar a cabo teniendo en cuenta las perspectivas teóricas de la segmentación y del capital humano. En este sentido, ninguna de las dos teorías presupone diferencias por sexo. Por un lado, asumir la dualidad del mercado de trabajo español implica aceptar que el mercado está dividido en dos segmentos y que dentro de cada uno las normas que rigen son distintas. Las diferencias entre estar en el segmento primario o secundario pueden suponer, por ejemplo, una mayor o menor estabilidad laboral (tipo de contratación, grado de rotación entre empleos...). Por otra parte, la segunda perspectiva teórica explica que el capital humano de los inmigrantes, por el hecho 
de haberlo adquirido en su país de origen, no ofrece el mismo rendimiento en el mercado de trabajo que el de los nativos. Todo ello explicaría la fuerte segregación ocupacional de los inmigrantes al entrar al mercado de trabajo - a causa de la devaluación de su capital humano-, pero también el menor rendimiento del nivel educativo y el tiempo en el mercado de trabajo en comparación con los españoles una vez ocupados - debido a encontrarse en el segmento secundario-.

La segregación sectorial de los inmigrantes afecta a todos por igual, tanto a mujeres como a varones. La mayoría de los trabajadores extranjeros están sobrerrepresentados en ocupaciones caracterizadas no sólo por su baja cualificación, sino también - y lo que es más importante - por ofrecer una menor estabilidad. La protección que reciben los trabajadores del segmento secundario es menor que la que disfrutan los del segmento primario (ocupaciones medias y altas). La inestabilidad debería pronunciarse en fases de cambio de ciclo, por ello, se prevé que el inicio de la crisis tenga un efecto similar sobre el nivel de ocupación de mujeres y hombres inmigrantes. En el caso de las mujeres españolas, por el hecho de no estar concentradas en las ocupaciones más bajas de la estructura ocupacional, se espera que el impacto de la recesión no les afecte tanto. Por tanto, la primera hipótesis de este trabajo es que el nivel de ocupación de las mujeres inmigrantes sufre desde el inicio de la crisis una caída similar a la de los inmigrantes varones, pero mayor que la de las mujeres españolas.

Por otra parte, se quiere estudiar la evolución de la ocupación de las mujeres inmigrantes teniendo en cuenta el capital humano. Los inmigrantes en general pueden experimentar una devaluación de su capital humano en dos sentidos: por el hecho de ubicarse en las peores ocupaciones - en el segmento secundario el rendimiento del capital humano es menor-y por su escaso capital humano específico del país de acogida, ya que la mayoría fue adquirido en su país de origen. Por ello, se espera que para grupos semejantes de nivel educativo y experiencia laboral, las diferencias entre mujeres y varones inmigrantes sean de nuevo pequeñas. En cambio, teniendo en cuenta que una proporción mayor de españolas ocupan puestos de trabajo de cualificación media y alta (segmento primario), se prevé que desde la recesión las mujeres españolas con mismo capital humano que las extranjeras experimenten una caída de la ocupación más suave. Estas diferencias se espera que sean mayores entre niveles educativos altos. Las mujeres españolas más cualificadas, cuyo capital humano no está expuesto a una posible devaluación por emigrar a otro país, deberían ocupar puestos de trabajo más afines a su formación que las mujeres inmigrantes con la misma cualificación, la mayoría de ellas posiblemente concentradas en peores ocupaciones. En cambio, es más probable que españolas e inmigrantes con niveles educativos bajos coincidan en ocupaciones de baja cualificación, acordes en ambos casos a su capital humano. Como segunda hipótesis se plantea que cuando el capital humano es el mismo, las tasas de empleo de las mujeres inmigrantes sufren caídas similares a la de sus compatriotas varones. En comparación con españolas con su mismo capital humano, se espera que las mujeres inmigrantes experimenten caídas más fuertes. No obstante, las diferencias entre españolas y extranjeras deberían reducirse conforme menor sea el capital humano. Esta segunda hipótesis se 
comprobará por separado para el nivel educativo y para el tiempo en el mercado laboral.

Estas dos hipótesis apuntan hacia dos direcciones. Por un lado, hacia la segregación ocupacional que sufren en general las mujeres y los hombres inmigrantes, la cual debería perjudicarles en términos de estabilidad laboral, más todavía en periodos recesivos. Por otro, hacia el capital humano de los trabajadores extranjeros, el cual ha sido adquirido en el periodo previo a la emigración y, por ello, ofrece un rendimiento inferior en el mercado de trabajo. A partir de estos dos enfoques teóricos se debería poder explicar las semejanzas y diferencias del empleo de las mujeres inmigrantes respecto a los varones inmigrantes y las mujeres españolas.

\section{Datos, variables y técnicas}

Los datos utilizados en este trabajo pertenecen a la Encuesta de Población Activa (EPA), realizada por el Instituto Nacional de Estadística desde 1964 y con carácter trimestral desde 1975. La EPA es una encuesta con una muestra de 60.000 hogares y, por tanto, con información de alrededor de 180.000 individuos. Se trata de la principal fuente de información estadística sobre el mercado de trabajo en España. Los datos empleados en este artículo pertenecen al periodo comprendido entre el primer trimestre de 2000 y el segundo trimestre de 2010. De este modo se recoge la evolución desde la entrada masiva de inmigrantes a España hasta la actualidad, pasando por el inicio de la actual crisis económica a finales de 2007. Para que el tamaño muestral aumente, y con ello la fiabilidad de los resultados, los cuatro trimestres de cada año de la EPA han sido agrupados (Garrido y Toharia, 2004).

A lo largo de este trabajo se van a comparar a mujeres y varones inmigrantes entre sí, pero también a la población extranjera con la nativa, en concreto a las mujeres inmigrantes con las españolas. Para ello, es necesario crear grupos de análisis lo más homogéneos posibles. La población inmigrante en España responde a unas características sociodemográficas concretas, con casi un $60 \%$ de su población en edades comprendidas entre los 25 y los 44 años, y la mayoría de ellos inserta en el mercado laboral (Pérez Infante, 2008). Los extranjeros residentes en España con edades próximas al final de la vida laboral suelen ser personas que disfrutan ya de su prejubilación, principalmente procedentes de países de la Unión Europea previa a la ampliación de 2004 (UE-15) ${ }^{4}$. Por ello para homogeneizar los dos grupos, en vez de trabajar con toda la población en edad de trabajar (16-64 años), se ha optado por ceñir el estudio a la población comprendida entre los 16 y los 49 años, con la exclusión de quienes afirman estar estudiando.

${ }^{4}$ Los Estados miembros que componen la Unión Europea antes de la ampliación de 2004 son Alemania, Austria, Bélgica, Dinamarca, España, Finlandia, Francia, Grecia, Irlanda, Italia, Luxemburgo, Países Bajos, Portugal, Reino Unido y Suecia. 
Las tasas que se van a estudiar a lo largo del periodo 2000-2010 son la de empleo y la de paro ${ }^{5}$. La primera se calcula dividiendo el número de personas ocupadas entre el total de la población comprendida (en este caso todos los individuos entre 16 y 49 años). La tasa de paro, por otra parte, se extrae dividiendo el número de personas desocupadas entre la población de referencia activa (que está trabajando o que se encuentra desempleada y busca empleo). Cada tasa se calcula respecto a una población de referencia distinta - total de la población para la primera y población activa para la segunda-, por tanto, se advierte que la tasa de paro no es la misma que la de empleo invertida.

Respecto a las variables explicativas, para estudiar el efecto de la nacionalidad se ha escogido una clasificación de cuatro grupos. De ella no están incluidos los españoles nacidos en el extranjero, los españoles con doble nacionalidad y los nacionales procedentes de Asia, ya que todos ellos presentan erraticidad en su evolución debido a la escasez de casos. No obstante, cuando el análisis distingue únicamente dos grupos - extranjeros y españoles-, los nacionales de Asia sí son incluidos en el conglomerado de extranjeros. Por otra parte, los nacionales procedentes de la UE-15 son excluidos de la muestra, ya que sus tasas de empleo y paro, tanto de hombres como de mujeres, son a lo largo de todo el periodo estudiado muy parecidas a la de los españoles (Muñoz Comet, 2011). De este modo, el análisis de la población extranjera se va a centrar en la inmigración económica. Los cuatro grupos son los siguientes:

- Españoles nacidos en España

- Nacionales de América Central y del Sur

- Nacionales de Europa de fuera de la UE-15 (también referidos como europeos del Este)

- Nacionales de África

La segunda variable independiente del artículo es el nivel educativo alcanzado, agrupado en las siguientes cuatro categorías:

- Nivel 1 - Estudios primarios o menos (sin estudios, analfabetos y primaria)

- Nivel 2 - Estudios secundarios inferiores (EGB2, Bachillerato elemental y ESO)

- Nivel 3 - Estudios secundarios superiores (FP y Bachillerato superior)

- Nivel 4 - Estudios universitarios (universidad media y superior)

Por último, se va a estudiar el efecto del tiempo en el mercado de trabajo, es decir, la experiencia laboral potencial. Esta variable, que no se recoge directamente en la EPA, se ha calculado de forma diferente para españoles y para extranjeros. Para los primeros, la edad laboral comienza en el momento en que se abandona el siste-

\footnotetext{
${ }^{5}$ También nos referiremos a la tasa de empleo como el nivel de ocupación.
} 
ma educativo reglado. En el caso de los inmigrantes, se considera que su inserción en el mercado de trabajo español arranca el mismo año de llegada al país de acogida, siempre y cuando hayan acabado de estudiar en su país de origen. En los que casos en los que continúan sus estudios al llegar a España, su edad laboral se calcula de la misma forma que para la población autóctona ${ }^{6}$.

Cuando se estudia la integración laboral de los inmigrantes, el uso de datos transversales puede ser problemático y conducir a ciertos sesgos. La detección de cambios en la cualificación de las diferentes cohortes de llegada, así como de aquellos inmigrantes que retornan a sus países, puede llevarse a cabo mediante datos longitudinales (Borjas, 1985; Borjas y Bratsberg, 1996). Obviar cualquiera de estas dos posibilidades podría llevar a conclusiones erróneas sobre el proceso de asimilación de los inmigrantes. Puesto que los datos utilizados en este trabajo no son de carácter longitudinal, los sesgos producidos por la migración de retorno resultan complicados de controlar. Sin embargo, la construcción de cohortes ficticias sí que permite observar la cualificación de las diferentes olas de llegada. A partir de diferentes cohortes en distintos años, se pueden crear cohortes ficticias con individuos que, aunque no son los mismos, pertenecen a la misma cohorte y poseen las mismas características sociodemográficas.

El efecto del tiempo en el mercado de trabajo se va a calcular, por esta razón, para dos cohortes ficticias distintas, cuya principal diferencia entre ellas será la experiencia laboral potencial que habrán acumulado justo antes de comenzar la crisis en 2008. La cohorte de entrada al mercado laboral 2000-2003 agrupa a aquellos que llegaron a España durante la expansión del ciclo económico, con un intenso ritmo de crecimiento del empleo. Este grupo pudo disfrutar de un periodo de estabilidad laboral antes de que la recesión comenzara. Por otra parte, la cohorte 2006-2007 recoge un cambio en la composición nacional de los flujos, con un mayor protagonismo de las llegadas procedentes de Europa del Este y países latinoamericanos, hasta el momento con menos presencia en el país. La llegada de esta cohorte coincide con el año previo al inicio de la crisis, cuando el mercado de trabajo de los varones inmigrantes ya comienza a mostrar síntomas de saturación (Garrido y Miyar, 2008).

${ }^{6}$ Se advierte de que en este trabajo se va a estudiar el efecto del tiempo en el mercado de trabajo, y no el tiempo de residencia o el año de llegada al país de destino. Por ello, el tiempo laboral potencial se calcula teniendo en cuenta el momento en que se termina de estudiar. De este modo, para un extranjero que ha finalizado sus estudios tras emigrar, su tiempo de residencia en España y su edad laboral no coinciden. 


\section{Resultados}

La primera hipótesis se centra en la evolución de la ocupación una vez comenzada la crisis. Uno de los aspectos más reseñables a primera vista es que hasta el inicio de la recesión económica las tasas de las mujeres inmigrantes son bastante diferentes de las de los inmigrantes varones. En general hasta el año 2008 la tasa de empleo de los varones es claramente superior a la de las mujeres. En cuanto a la tasa de paro, la de los hombres es menor, aunque en este caso las diferencias respecto a las mujeres no son tan grandes. La razón por la que hay más diferencias entre sexos en las tasas de empleo es porque se está teniendo en cuenta a toda la población y no sólo a la población activa. Tomar como referencia a una u otra población ofrece imágenes diferentes. Un ejemplo claro es el de las mujeres africanas, quienes experimentan unas tasas de actividad muy bajas. La brecha de las africanas respecto al resto de nacionalidades, siempre muy amplia, es algo menor cuando se presta atención a la tasa de paro.

El nivel de ocupación de las mujeres inmigrantes difiere hasta 2007 dependiendo de la nacionalidad (Gráfico 1). En general todos los grupos experimentan una mejoría de entre cinco y diez puntos porcentuales a lo largo de este periodo, aunque son las latinoamericanas las que parten de una tasa de empleo más elevada. Las africanas, por el contrario, se desmarcan del resto de grupos con mucha más distancia. Al finalizar este periodo de crecimiento, las tasas se estabilizan. El nivel de ocupación de las latinoamericanas es el único que sufre una caída más fuerte que el resto. Con un descenso de hasta 14 puntos porcentuales, la ventaja que disfrutaban en los años previos frente a españolas y europeas de fuera de la UE-15 desaparece. En el caso de los varones, el nivel y la evolución de sus tasas de empleo hasta 2007 son muy parecidos en todas las nacionalidades, a excepción de los africanos (Gráfico 1). Durante ese periodo el nivel de ocupación está en torno al $85 \%$, con un leve crecimiento hacia el 90\%. En 2008 las tasas de empleo caen de forma generalizada, aunque este descenso ya lo comienzan a experimentar latinoamericanos, europeos del Este y africanos un año antes. A partir de ese año las tasas de todos los grupos decrecen, aunque a ritmos diferentes. Se distinguen tres grupos en función de su ritmo de caída. El descenso más fuerte lo sufren los africanos, quienes pasan de tener un $79 \%$ en 2007 a un $50 \%$ en 2010 . Los latinoamericanos y los europeos del Este conforman un segundo grupo, con una pérdida en ese mismo periodo próxima a los 23 puntos porcentuales. En tercer lugar se encuentran los españoles, cuya caída es de 11 puntos. 
Gráfico 1. Tasa de empleo por sexo y nacionalidad (16-49 años)

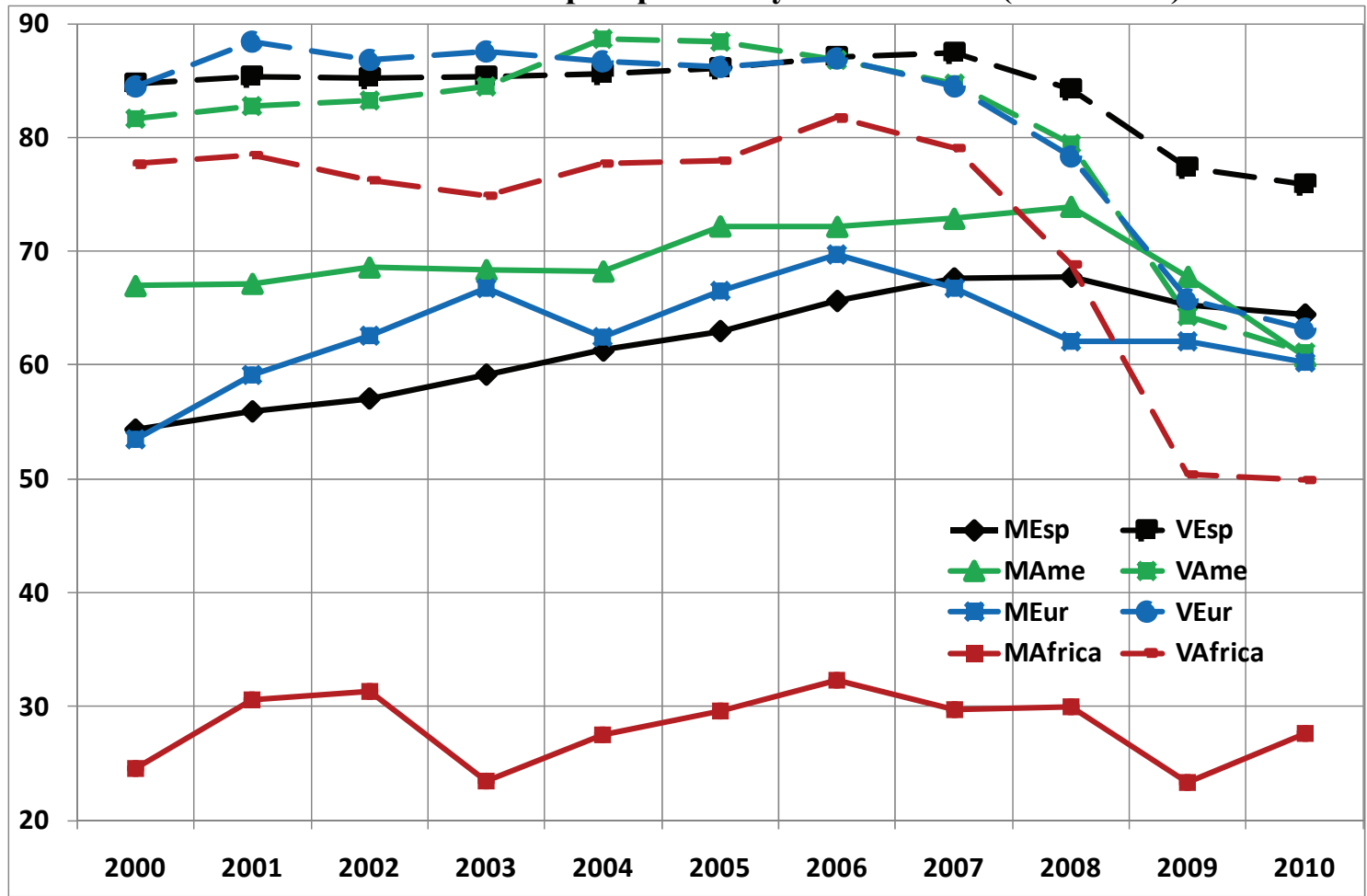

Fuente: elaboración propia a partir de la EPA (I/2000-II/2010)

Respecto a las tasas de paro, entre las mujeres se observan algunos aspectos diferentes en comparación con el empleo. En el año 2000 todos los grupos mantienen unas tasas muy parecidas (alrededor del 20\%), excepto africanas, con una tasa del $39 \%$ (Gráfico 2). Estas últimas se mantienen alejadas del resto de grupos a lo largo de todo el periodo de estudio, a una distancia de entre 20 y 30 puntos porcentuales. Hasta 2005 la evolución general es de caída de la tasa de paro, año en el que prácticamente convergen todos los grupos con un tasa en torno al $12 \%$. Tras un año de estabilidad, las tasas comienzan a crecer lentamente, distanciándose ligeramente unas de otras. En 2010 la tasa de paro de estos grupos se mueve entre el 20 y el 25 por ciento, siendo la de las españolas la más baja.

La evolución de la tasa de paro de los varones entre 2000 y 2010 es prácticamente similar a la observada en la tasa de empleo. Hasta el inicio de la crisis todos los grupos muestran unas tasas muy parecidas, a excepción de los africanos. A partir de 2007 todos los grupos comienzan a experimentar un aumento de la tasa de paro, aunque a ritmos distintos. Se distinguen de nuevo los tres grupos mencionados anteriormente (Gráfico 2). 
Gráfico 2. Tasa de paro por sexo y nacionalidades (16-49 años)

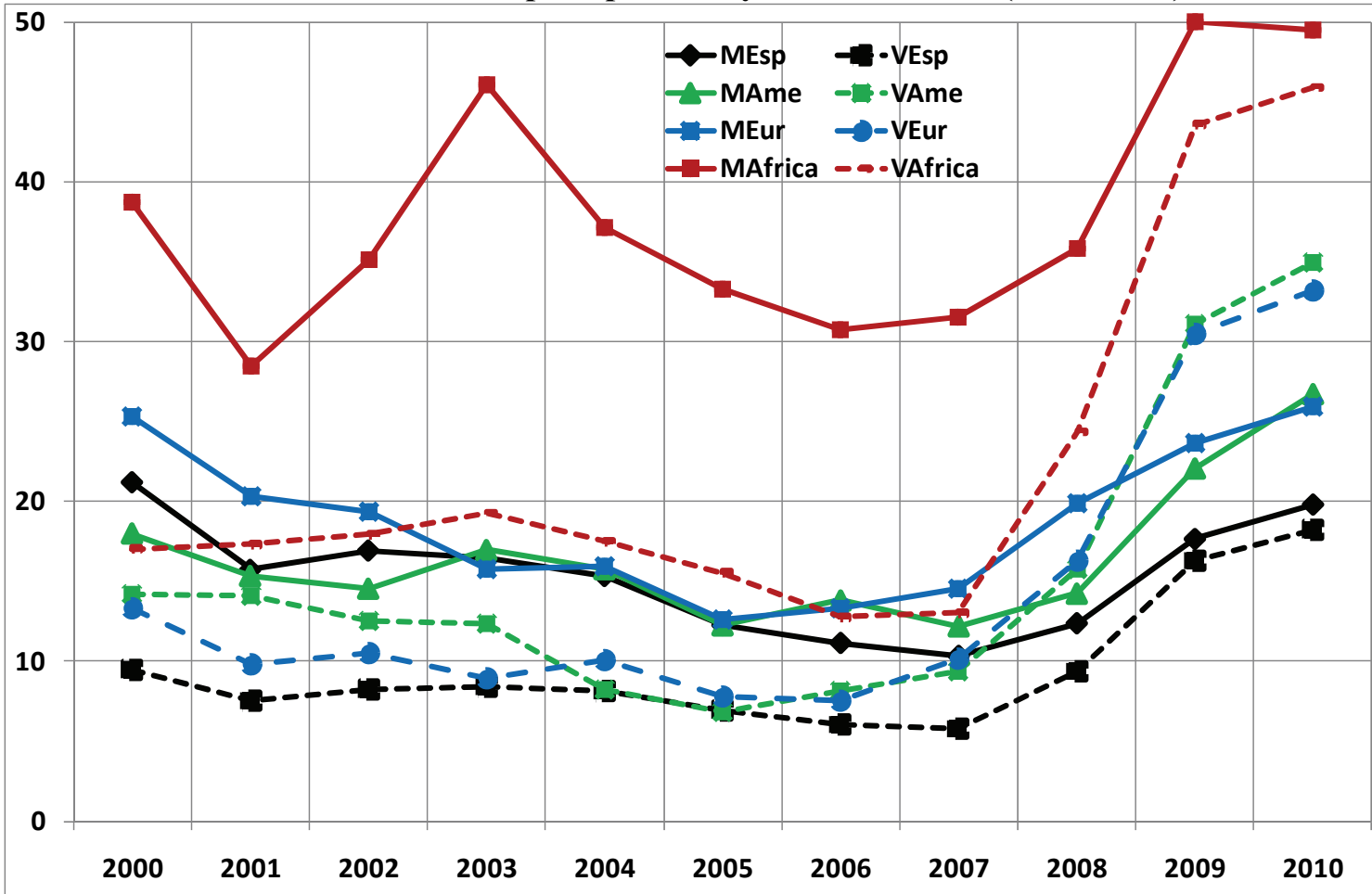

Fuente: elaboración propia a partir de la EPA (I/2000-II/2010)

La primera hipótesis predecía que, en términos generales, la evolución de las tasas de mujeres y hombres inmigrantes sería bastante parecida desde el inicio de la crisis. También se preveía que las mujeres españolas sufrieran menos pérdidas en su ocupación que las mujeres inmigrantes. En lo que respecta a la comparación entre sexos, no se confirma que el efecto de la crisis haya sido igual para mujeres inmigrantes que para sus connacionales varones. Las extranjeras, en concreto las latinoamericanas y sobre todo las europeas del Este, soportan bastante mejor el impacto de la crisis que sus compatriotas. Al final del periodo estudiado las tasas de empleo de los hombres convergen con las de ellas, mientras que las tasas de paro incluso las superan. En el caso del colectivo africano, la caída de la tasa de empleo es mayor entre los varones, y cuando nos fijamos en la tasa de paro, su tasa y la de las mujeres africanas están incluso cerca de converger en 2010. Por otra parte, cuando comparamos a mujeres inmigrantes y españolas, las predicciones son algo más acertadas. En general todos los grupos gozan de cierta estabilidad en lo que respecta a sus tasas de empleo. Habría que destacar el caso de las mujeres de Europa del Este, cuyo nivel de ocupación, a pesar de experimentar una caída significativa en 2006 y 2007, a partir de la crisis mantiene una evolución prácticamente igual 
que las mujeres españolas. No obstante, las tasas de paro de todos los grupos crecen a partir de 2007, aunque en menor medida para las autóctonas.

\subsection{Nivel educativo}

La segunda hipótesis del trabajo hacía referencia al rendimiento del capital humano a partir del cambio de ciclo económico, y para ello nos centrábamos en el nivel educativo y en el tiempo en el mercado de trabajo. En cuanto al primero de ellos, vemos de nuevo cómo las tasas de empleo de mujeres y hombres inmigrantes difieren bastante a lo largo de todo el periodo estudiado (Tabla 1). En el grupo con estudios de primaria o menos, la tasa de empleo de las mujeres inmigrantes crece hasta 2007, año a partir del cual su tasa sufre una caída de 11 puntos porcentuales hasta 2010. En el caso de los inmigrantes varones con primaria o menos, su evolución hasta el inicio de la crisis es más estable y su caída entre 2007 y 2010 es de 27 puntos porcentuales. En el grupo de estudios secundarios inferiores, las mujeres inmigrantes repiten el mismo patrón que las del nivel educativo anterior, crecimiento hasta 2007 y después descenso, aunque su caída es más suave (cuatro puntos). La evolución de los inmigrantes varones con estudios secundarios inferiores se asemeja a la de los que tienen primaria o menos. El descenso de su tasa de empleo a partir de la recesión es de 24 puntos porcentuales.

Las mujeres inmigrantes con estudios secundarios superiores también experimentan un crecimiento desde 2000 hasta 2007 y después un descenso de seis puntos, bastante parecido al que tienen las mujeres con un nivel educativo secundario inferior. En el caso de los inmigrantes varones con estudios secundarios superiores, la caída de su tasa a partir de 2007 continúa siendo muy acentuada, de 21 puntos hasta 2010. Por último, en el grupo con nivel de estudios universitario, la tasa de empleo de las mujeres inmigrantes prácticamente se mantiene igual a lo largo de la crisis. Por el contrario, los varones inmigrantes con su mismo nivel de estudios sufren una caída de 11 puntos, esta vez algo menos fuerte que la del resto de compatriotas varones con nivel de estudios inferior. Ante estos resultados se puede afirmar que entre los extranjeros, poseer un nivel de estudios alto implica sufrir menos pérdidas en el nivel de ocupación. Sin embargo, mientras que para las mujeres inmigrantes con nivel educativo universitario la llegada de la crisis apenas afecta a su tasa de empleo, entre los hombres con ese mismo nivel de estudios su tasa sufre todavía una caída muy fuerte. 
Tabla 1. Tasa de empleo por nivel educativo (16-49 años)

\begin{tabular}{|c|c|c|c|c|c|c|c|c|c|c|c|c|}
\hline ESTUDIOS & 2000 & 2001 & 2002 & 2003 & 2004 & 2005 & 2006 & 2007 & 2008 & 2009 & 2010 & $2010-2007$ \\
\hline \multicolumn{13}{|c|}{ Primaria o menos } \\
\hline Extranjeras & 36 & 47 & 53 & 45 & 47 & 52 & 51 & 51 & 49 & 44 & 40 & -11 \\
\hline Extranjeros & 81 & 80 & 78 & 78 & 83 & 82 & 83 & 81 & 69 & 55 & 54 & -27 \\
\hline Españolas & 36 & 37 & 38 & 38 & 39 & 40 & 42 & 42 & 43 & 39 & 38 & -4 \\
\hline \multicolumn{13}{|c|}{ Secundarios inferiores } \\
\hline Extranjeras & 53 & 59 & 61 & 64 & 61 & 61 & 64 & 61 & 64 & 59 & 57 & -4 \\
\hline Extranjeros & 87 & 82 & 85 & 85 & 85 & 83 & 84 & 80 & 75 & 57 & 55 & -24 \\
\hline Españolas & 48 & 49 & 49 & 51 & 52 & 52 & 55 & 58 & 57 & 53 & 53 & -5 \\
\hline \multicolumn{13}{|c|}{ Secundarios superiores } \\
\hline Extranjeras & 60 & 61 & 65 & 68 & 68 & 71 & 72 & 71 & 69 & 64 & 65 & -6 \\
\hline Extranjeros & 80 & 82 & 82 & 84 & 87 & 87 & 88 & 86 & 82 & 68 & 66 & -21 \\
\hline Españolas & 63 & 64 & 65 & 66 & 68 & 69 & 71 & 72 & 72 & 70 & 67 & -5 \\
\hline \multicolumn{13}{|c|}{ Universitarios } \\
\hline Extranjeras & 69 & 66 & 69 & 63 & 62 & 68 & 71 & 69 & 68 & 67 & 68 & -1 \\
\hline Extranjeros & 78 & 89 & 84 & 88 & 87 & 88 & 84 & 85 & 80 & 69 & 72 & -13 \\
\hline Españolas & 76 & 77 & 78 & 80 & 81 & 82 & 84 & 86 & 85 & 83 & 82 & -4 \\
\hline
\end{tabular}

Fuente: elaboración propia a partir de la EPA (I/2000-II/2010)

Hemos visto que entre las mujeres inmigrantes, cada nivel educativo experimentaba al inicio de la crisis tasas de empleo diferentes, pero las caídas que sufrían a partir de ese momento eran suaves y menores conforme mayor era el nivel de estudios. Entre las mujeres españolas las tasas de empleo también experimentan niveles diferentes a lo largo de todo el periodo en función del nivel educativo. De hecho, las tasas de las españolas son más bajas que las de las extranjeras en los dos niveles más bajos y sólo son parecidos en el nivel secundario superior. Es en el grupo de universitarias donde la tasa de empleo de las españoles supera con más margen a la de las extranjeras, tanto antes como durante la recesión económica. Una peculiaridad de las españolas es que la caída de su tasa a partir de la crisis es similar en todos los grupos educativos. Parten de niveles de ocupación diferentes, pero todos los niveles educativos caen entre cuatro y cinco puntos porcentuales. Por lo tanto, parece que entre las mujeres extranjeras la posesión de un nivel de estudios inferior o superior es más importante para frenar el efecto de la crisis que para las mujeres españolas.

En lo que respecta al nivel educativo, la segunda hipótesis no se confirma. Por un lado, las semejanzas por sexo no se producen. A partir de 2007 las mujeres inmigrantes ven caer sus tasas mucho menos que los inmigrantes varones con su mismo nivel de estudios. Respecto a las mujeres españolas, observamos que las diferencias en las pérdidas en el nivel de ocupación se encuentran en los grupos educativos más alto y más bajo, pero justo al contrario de cómo se predecía. Mien- 
tras que las mujeres inmigrantes con nivel educativo primario o menos tienen una caída más acentuada que las españolas con ese mismo nivel, en el grupo de universitarias las pérdidas son menores entre inmigrantes que entre españolas. La clave parece estar en que para las mujeres españolas la llegada de la crisis afecta a todos los grupos educativos por igual, mientras que entre las inmigrantes un nivel de estudios más elevado se relacionado con una caída de la tasa de empleo más baja. No obstante, el nivel de ocupación en el nivel educativo universitario es claramente mayor entre españolas que entre extranjeras. Es decir, aunque las españolas con estudios universitarios vean caer su nivel de ocupación en mayor medida que las extranjeras con su mismo nivel educativo, las españolas disfrutan a lo largo de todo el periodo de unas tasas de empleo superiores.

\subsection{Tiempo en el mercado de trabajo español}

El segundo factor relacionado con el capital humano es la influencia del tiempo en el mercado de trabajo español. En los tres grupos la tasa de empleo de la cohorte de 2000-2003 experimenta crecimiento los primeros años, siendo la de las mujeres españolas — quienes parten del nivel más bajo - la que muestra un incremento más pronunciado (Gráfico 3). Los inmigrantes varones son los primeros en ver frenar ese crecimiento, en su cuarto año, mientras que las mujeres inmigrantes y españolas mantienen esa tendencia hasta el quinto. Para algunas personas de la cohorte 20002003 el quinto año representa el comienzo de la crisis. A partir de ese momento la evolución es diferente según el grupo. La tasa de empleo de los inmigrantes varones sufre una fuerte caída de 26 puntos porcentuales desde el cuarto hasta el noveno año en el mercado de trabajo. En este mismo periodo, la tasa de empleo de las mujeres inmigrantes sufre un desplome menos fuerte, de 15 puntos. En el caso de las mujeres españolas, su tasa decrece tan sólo tres puntos. Al fijarnos en la cohorte 20062007, los varones inmigrantes y las mujeres españolas experimentan al principio la misma progresión que la cohorte anterior (2000-2003), pero después del primer y segundo año, respectivamente, sus tasas se frenan. La tasa de empleo de los varones inmigrantes, después de su primer año en el mercado de trabajo, cae 11 puntos en los dos siguientes años. En el caso de las mujeres españolas, la caída después de su segundo año en el mercado laboral es pequeña (tres puntos). Frente a estas dos evoluciones, la tasa de empleo de las mujeres inmigrantes no deja de crecer en ningún momento. Es decir, la llegada de la crisis para la cohorte más joven de mujeres inmigrantes no significa, a diferencia de los otros dos grupos, una pérdida del nivel de ocupación.

Si comparamos las dos cohortes estudiadas entre sí se pueden extraer algunas conclusiones. Por un lado, que un mayor tiempo en el mercado laboral sólo parece ser efectivo ante la crisis para las mujeres españolas. Tanto hombres como mujeres inmigrantes que entraron al mercado de trabajo entre 2000 y 2003 tienen unas tasas de empleo al final del periodo estudiado (año 2009) similares que los que entraron entre 2006 y 2007 . El caso más claro de la no rentabilidad de un mayor tiempo en el mercado de trabajo sería el de la cohorte de 2000-2003 de varones inmigrantes. La caída que experimentan es tan fuerte que terminan prácticamente igualando el nivel 
de ocupación de la otra cohorte más joven, incluso sufriendo ésta también pérdidas a partir de su segundo año. A diferencia de ellos, en la cohorte 2000-2003 de mujeres inmigrantes la caída no es tan fuerte como entre los varones. No obstante, el nivel de ocupación de las dos cohortes de mujeres extranjeras también se iguala en su último año en el mercado de trabajo. Lo peculiar de este caso, sin embargo, es que la convergencia de las dos cohortes se debe al crecimiento ininterrumpido de la tasa de empleo de las inmigrantes que acceden al mercado entre 2006-2007.

\section{Gráfico 3. Tasa de empleo por tiempo potencial en el mercado de trabajo y} cohortes de entrada (16-49 años)

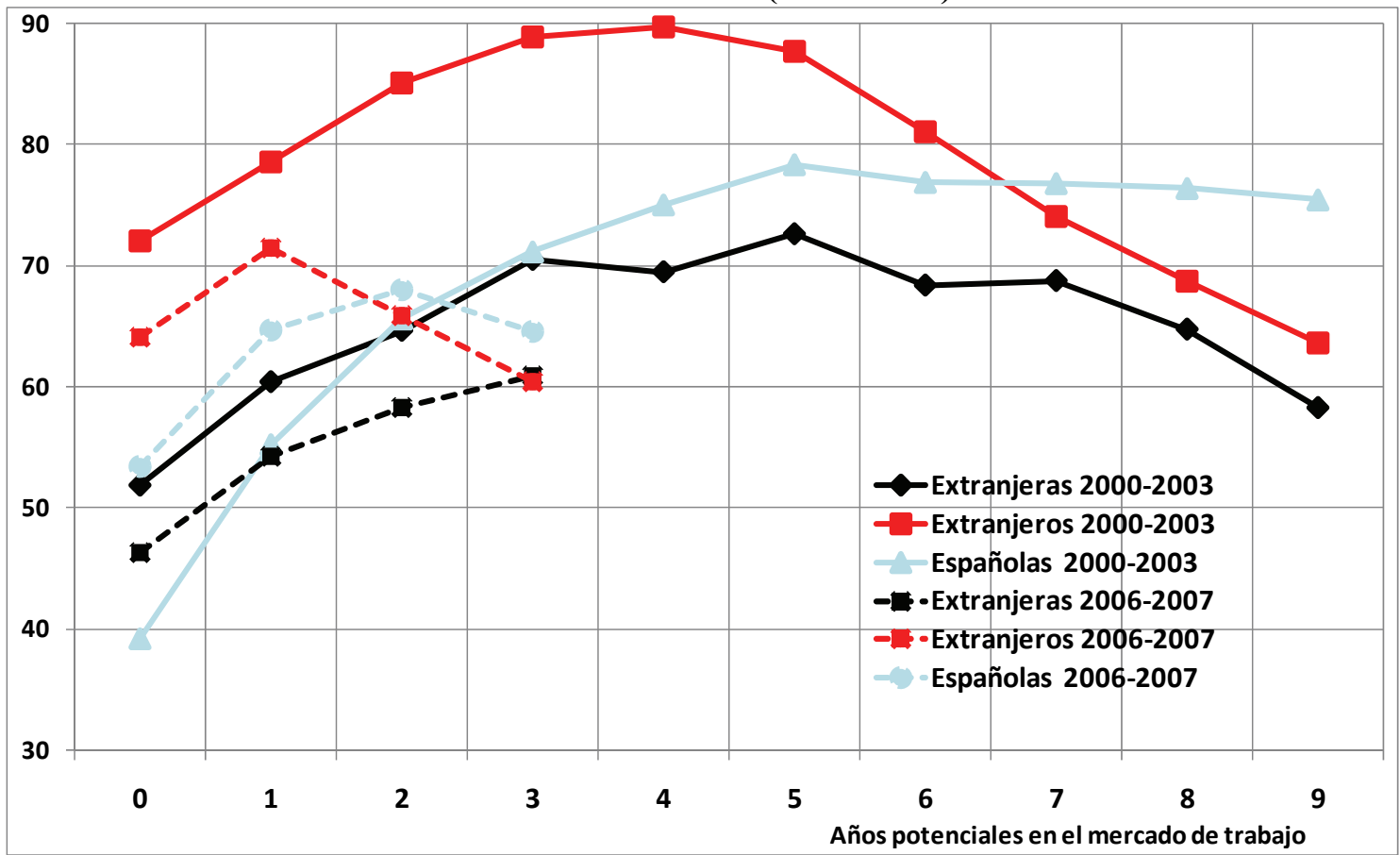

Fuente: elaboración propia a partir de la EPA (I/2000-IV/2009)

Por todo ello, en lo que respecta al tiempo en el mercado laboral se confirma parcialmente la segunda hipótesis. Al comparar mujeres y hombres inmigrantes, la evolución de sus tasas a partir de la crisis es sólo semejante en la cohorte más antigua (2000-2003), aunque la caída que ambos grupos experimentan es más intensa entre los varones. La tendencia, sin embargo, no es la misma al observar la cohorte más joven (2006-2007). Mientras que los inmigrantes varones ven caer su tasa de empleo a partir del segundo año en el mercado laboral, el nivel de ocupación de las mujeres extranjeras no para de crecer. Aunque para ninguno de los dos grupos un mayor tiempo en el mercado de trabajo se traduce en una mayor resistencia 
contra la crisis, las mujeres inmigrantes que accedieron al mercado justo antes del comienzo de la recesión parecen repetir la misma evolución de crecimiento durante los primeros años que la cohorte más antigua. En cuanto a las mujeres españolas, el nivel de ocupación para la cohorte 2000-2003 se mantiene estable tras los primeros años de crecimiento ininterrumpido. Es por tanto el único grupo que parece rentabilizar claramente su tiempo en el mercado laboral en comparación con la cohorte más joven de su mismo grupo. Para las españolas con menos tiempo en el mercado de trabajo, la evolución de su tasa de empleo es más parecida a la de las mujeres inmigrantes de su misma cohorte, aunque experimentan una pequeña caída en su último año en el mercado laboral que las extranjeras no sufren.

\section{Conclusiones}

En este artículo se ha realizado un análisis descriptivo de la ocupación de las mujeres inmigrantes desde el año 2000 hasta el año 2010. Para interpretar esa evolución se han utilizado dos enfoques teóricos, la segmentación del mercado de trabajo español y la importancia del capital humano en la integración laboral. El objetivo del trabajo era conocer el rendimiento que ofrece el capital humano de las mujeres inmigrantes una vez comenzada la crisis. Es decir, si un nivel educativo alto y un mayor tiempo en el mercado de trabajo se asocian con caídas más pequeñas de la ocupación. Para ello, la evolución de empleo de las mujeres extranjeras ha sido comparado con la de los varones inmigrantes y la de las mujeres españolas.

Partiendo de los dos enfoques teóricos se definían las hipótesis del estudio. En primer lugar, que las tasas de empleo de las mujeres inmigrantes sufrirían a partir de la crisis una misma caída que las de los varones inmigrantes. La sobrerrepresentación de los trabajadores extranjeros en las ocupaciones con menor estabilidad laboral debería afectarles con mayor fuerza durante un periodo de recesión. Dentro de esta primera hipótesis se añadía que la tasa de empleo de los inmigrantes, para ambos sexos, sufriría una caída más grande que la de las españolas. Estas, a diferencia de las mujeres extranjeras, no sufren una concentración sectorial tan fuerte y la presencia en las ocupaciones medias y altas es mayor. Ello debería traducirse en una mayor estabilidad durante el cambio de ciclo económico. Por otra parte, la segunda hipótesis predecía que las diferencias entre las tasas de empleo de mujeres inmigrantes y españolas serían mayores cuanto mayor fuera su capital humano. Un nivel educativo alto y un mayor tiempo en el mercado laboral deberían ser más rentables para españolas que para mujeres inmigrantes, puesto que estas últimas están fuertemente sobrerrepresentadas en el segmento secundario. Las diferencias, en cambio, deberían ser menores entre grupos de mujeres inmigrantes y españolas con un capital humano menor, ya que es más probable que coincidan en las mismas ocupaciones y la penalización por estar en el segmento secundario sea similar en ambos grupos.

Los resultados muestran que hasta el inicio de la crisis las tasas de empleo de mujeres y varones marcaban niveles muy diferentes. Si nos centramos en lo que 
ocurre a partir de la recesión, se observa que las mujeres inmigrantes sufren unas pérdidas en el nivel de ocupación mucho menores que los inmigrantes varones. La ventaja que disfrutaban los hombres frente a las mujeres hasta 2008 desaparece en 2010 y en algunos casos incluso se invierte (para las tasas de paro de latinoamericanas y europeas de fuera de la UE15). En comparación con las mujeres españolas, la evolución de las tasas de empleo son bastante similares, pero no en la tasa de paro, en la que las mujeres inmigrantes experimentan aumentos mayores. Tal vez sea importante mencionar que gran parte de la población procedente de Europa del Este pasó a ser comunitaria precisamente en el año 2007, lo que implica una posición legal mucho menos vulnerable en el mercado de trabajo frente a la de las extranjeras sometidas al régimen general de extranjería. Ello podría explicar porqué las tasas de las mujeres de fuera de la UE-15 muestran desde 2008 una evolución bastante parecida a las de las españolas. Todos estos resultados confirman parcialmente la primera hipótesis. Mientras que entre las mujeres y los hombres inmigrantes se hallan unas diferencias que no se presuponían, las mujeres españolas muestran más resistencia en comparación con las mujeres inmigrantes.

Respecto a la segunda hipótesis, se encuentran resultados interesantes. En lo referente al nivel educativo, las diferencias entre mujeres y hombres inmigrantes son notables, algo que tampoco se preveía. Las pérdidas de empleo a partir de la crisis entre las mujeres inmigrantes son en general bajas, bastante menores que las que sufren los varones inmigrantes. No obstante, en ambos grupos se aprecia una correspondencia positiva entre el nivel educativo y unas pérdidas menores. Frente a ello, las mujeres españolas muestran caídas bajas, pero muy parecidas en todos los niveles de estudios. La semejanza con las mujeres inmigrantes no se produce en el grupo educativo más bajo (segunda hipótesis), sino en los dos intermedios. En el nivel universitario las pérdidas son incluso menores entre extranjeras que entre españolas, aunque el nivel de ocupación a lo largo de todo el periodo estudiado es claramente superior para estas últimas. Finalmente, un mayor tiempo en el mercado de trabajo parece que sólo es eficaz ante la crisis entre las mujeres españolas. En general, los inmigrantes con más años en el mercado laboral no disfrutan caídas de sus tasas de empleo más suaves que los inmigrantes con menos tiempo. En el caso concreto de las mujeres inmigrantes con menos tiempo, el crecimiento de su tasa de empleo no remite en ningún momento, algo que no ocurre ni entre varones extranjeros ni entre españolas.

En vista de estos resultados, se podría decir que la influencia del capital humano puede estar condicionada por factores estructurales. Muestra de ello lo encontraríamos en el empleo de los inmigrantes varones, el cual da signos de saturación antes incluso del comienzo de la crisis. En este grupo el rendimiento de un capital humano alto es claramente menor que entre mujeres inmigrantes y españolas. Las extranjeras soportan el efecto de la crisis mucho mejor que sus compatriotas varones y el nivel educativo parece jugar un papel más importante, teniendo en cuenta que el nivel de ocupación de las extranjeras con estudios universitarios se mantiene estable. En lo que concierne al tiempo en el mercado de trabajo, las cohortes más antiguas de inmigrantes (2000-2003) sufren unas caídas en el empleo que las igualan con las 
cohortes más recientes (2006-2007). No obstante, es interesante observar que entre las mujeres inmigrantes que acceden al mercado laboral justo antes de la crisis, su tasa de empleo continua creciendo incluso una vez comenzada la recesión. Esto puede ser un indicio de que los nichos laborales en los que se concentran las mujeres inmigrantes todavía ofrecen posibilidades pese a la crisis. En definitiva, la activación del capital humano podría estar condicionada por la saturación de los mercados específicos (diferencias entre mujeres y hombres inmigrantes) y por el segmento en el que se ubican los trabajadores (diferencias entre mujeres inmigrantes y españolas).

Una de las conclusiones de este trabajo es que el nivel de ocupación de las mujeres inmigrantes muestra más similitudes con el de las españolas que con el de sus connacionales varones. Los resultados de este artículo, no obstante, deberían profundizarse con otros análisis que permitan conocer el verdadero efecto del capital humano y de otros factores relacionados con la segmentación del mercado laboral. En este sentido sería interesante estudiar qué variables explican las diferencias entre mujeres inmigrantes y sus connacionales, así como en comparación con las mujeres españolas, en la probabilidad de estar ocupado. Descubrir hasta qué punto el capital humano de las extranjeras permanece devaluado desde su llegada a España puede resultar de gran interés, ya que de ello dependerá buena parte de sus posibilidades para mejorar su posición en el mercado de trabajo español.

\section{Bibliografía}

Amuedo Dorantes, C. y De la Rica, S. (2006). Labor market assimilation of recent immigrants in Spain. IZA Discussion Paper 2104.

Becker, G. S. (1999). Inversión en capital humano e ingresos. En L. Toharia (ed.), El mercado de trabajo. Teorías y aplicaciones (pp. 39-63). Madrid: Alianza Editorial.

Bernardi, F. y Garrido, L. (2008). Is there a new service proletariat? Post-industrial employment growth and social inequality in Spain. European Sociological Review, 24 (3), 299-313.

Bernardi, F, Garrido, L. y Miyar, M. (2011). The recent fast upsurge of immigrants in Spain and their employment patterns and occupational attainment. International Migration, 49 (1), 148-187.

Boisjoly, J. y Duncan, G. (1994). Job losses among Hispanics in the recent recession. Monthly Labor Review, 117, (6), 16-23.

Borjas, G. J. (1985). Assimilation, changes in cohort quality and the earnings of immigrants. Journal of Labor Economics, 3 (4), 463-489. 
Borjas, G. J. y Bratsberg, B. (1996). Who leaves? The outmigration of the foreignborn. The Review of Economics and Statistics, 78 (1), 165-176.

Cachón, L. (1997). Segregación sectorial de los inmigrantes en el mercado de trabajo en España. Cuadernos de Relaciones Laborales, 10, 49-73.

Cebolla, H. y González, A. (2008). La inmigración en España (2000-2007). De la gestión de los flujos a la integración de los inmigrantes. Madrid: Centro de Estudios Políticos y Constitucionales.

Chiswick, B. R. (1978). The effect of americanization on the earnings of foreignborn men. Journal of Political Economy, 86 (5), 897-921.

Chiswick, B. R. (1979). The economic progress of immigrants: some apparently universal patterns. En W. Fellner (ed.), Contemporary Economic Problems (pp. 357-399). Washington DC: American Enterprise Institute.

Chiswick, B. R., Cohen, Y. y Zach, T. (1997). The labor market status of immigrants: effects of the unemployment rate at arrival and duration of residence. Industrial and Labor Relations Review, 50 (2), 289-30.

Dustmann, C. (1994). Speaking fluency, writing fluency and earning of migrants. Journal of Population Economics, 7, 133-156

Dustmann, C. y Fabbri, F. (2003). Language proficiency and the labor market performance of immigrants in the United Kingdom. The Economic Journal, 113 (489), 695-717.

Fernández, C. y Ortega, C. (2008). Labor market assimilation of immigrants in Spain: employment at the expense of bad job-matches? Spanish Economic Review, 10 (2), 83-107.

Friedberg, R. (2000). You can't take it with you? Immigrant assimilation and the portability of human capital. Journal of Labor Economics, 18 (2), 221-251.

Garrido, L. (2008). La inmigración en España. En J. J. González y M. Requena (ed.). Tres décadas de cambio social en España (pp. 135-172). Madrid: Alianza Editorial.

Garrido, L. y Miyar, M. (2008). Dinámica laboral de la inmigración en España durante el principio del siglo XXI. Panorama Social, 8, 52-70. 
Garrido, L. Miyar, M. y Muñoz, J. (2010). La dinámica laboral de los inmigrantes en el cambio de fase del ciclo económico. Revista presupuesto y Gasto Público, $61,201-221$.

Garrido, L. y Rodríguez, J. C. (2011). Estructura ocupacional y carencias formativas en las empresas. Madrid: Forem.

Garrido, L. y Toharia, L. (2004). La situación laboral de los españoles y los extranjeros según la Encuesta de Población Activa. Economistas, 99, 74-88.

Garson, J. P. y Dumont, J. (2009). Crisis económica y migraciones: bases para la reflexión. En E. Aja, J. Arango y J. Oliver (ed.), La inmigración en tiempos de crisis (pp. 44-51). Barcelona: CIDOB.

Huguet, A. (1996). Dualidad en el mercado de trabajo español. Revista de Economía Aplicada, 11 (4), 81-104.

Iglesias, C. y Llorente, R. (2006). ¿Integración o segmentación laboral de los inmigrantes en el mercado de trabajo? Un análisis de cohortes. Instituto Universitario de Análisis Económico y Social, Documento de Trabajo 07/2006.

Kochhar, R. (2003). Jobs lost, jobs gained: the Latino experience in the recession and recovery. Pew Hispanic Center, Washington.

Kochhar, R. (2008). Latino workers in the ongoing recession: 2007 to 2008. Pew Hispanic Center, Washington.

Kogan, I. (2004). Last hired, first fired? The unemployment dynamics of male immigrants in Germany. European Sociological Review, 20 (5), 445-461.

Lam, K. C. y Liu, P. W. (2002). Earnings divergence of immigrants. Journal of Labor Economics, 20 (1), 86-104.

Mato, J. y Gutiérrez, R. (2010). Logros laborales de los inmigrantes económicos en España: el papel de la lengua española. En J. A. Alonso y R. Gutiérrez (dir.), Emigración y lengua. El papel del español en las migraciones internacionales (pp. 101-159). Madrid: Fundación Telefónica.

Muñoz Comet, J. (2011). Los efectos de la crisis en el empleo de los extranjeros. ¿Importan el nivel educativo y la edad laboral? Revista Española de Sociología (en prensa)

Pajares, M. (2009). Inmigración y mercado de trabajo. Informe 2009. Documentos del Observatorio Permanente de la Inmigración, 21. 
Papademetriou, D. G. y Terrazas, A. (2009). Immigrants and the current economic crisis: research evidence, policy challenges and implications. Migration Policy Institute.

Pérez Infante, J. I. (2008). Luces y sombras del mercado de trabajo en España: problemas, situación y tendencias. Economía industrial, 367: 35-49.

Piore M. J. (1999). Notas para una teoría de la estratificación del mercado de trabajo. En L. Toharia (ed.), El mercado de trabajo. Teorías y aplicaciones (pp. 193221). Madrid: Alianza Editorial.

Skyt, H., Rosholm, M., Smith, N. y Husted, L. (2001). Qualifications, discrimination or assimilation? An extended framework for analyzing immigrant wage gap. IZA Discussion Paper 365.

Weiss, Y., Sauer, R. y Gotlibovsky, M. (2003). Immigration, search and loss of skill. Journal of Labor Economics, 21 (3), 557-591. 